\title{
Maternal prenatal attitudes and postnatal breast-feeding behaviours in rural Bangladesh
}

\author{
Elaine A Yu ${ }^{1}$, Joan S Thomas ${ }^{1,2}$, Aatekah Owais ${ }^{1}$, Noor Tirmizi $^{2}$, ASG Faruque ${ }^{3}$, \\ Sumon K Das ${ }^{3}$, Shahed Rahman ${ }^{4}$, Benjamin Schwartz ${ }^{2}$ and Aryeh D Stein ${ }^{1, *}$ \\ 'Hubert Department of Global Health, Emory University, Rollins School of Public Health, 1518 Clifton Road NE, \\ Mailstop 1518-002-7BB, Atlanta, GA 30322, USA: ${ }^{2}$ CARE USA, Atlanta, GA, USA: ${ }^{3}$ iccdr, b International Centre \\ for Diarrhoeal Disease Research, Bangladesh), Clinical Sciences Division, Dhaka, Bangladesh: ${ }^{4}$ CARE Bangladesh, \\ Dhaka, Bangladesh
}

Submitted 9 September 2013: Final revision received 8 April 2014: Accepted 10 April 2014: First published online 19 May 2014

\begin{abstract}
Objective: To assess the relationships between maternal breast-feeding intention, attitudes, self-efficacy and knowledge at 7 months' gestation with exclusive or full breast-feeding at 3 months postpartum.

Design: Prospective cohort study with structured home interviews during pregnancy and 3 months after delivery.

Setting: Two rural sub-districts of Kishoreganj district, Bangladesh.

Subjects: Mother-infant dyads.

Results: Over $80 \%$ of 2178 pregnant women intended to exclusively breast-feed (EBF). Maternal positive attitudes, self-efficacy and knowledge about breastfeeding were positively associated with EBF intention (all $P<0 \cdot 05$ ). All mothers except one reported initiating breast-feeding and $99.6 \%$ of children were still breast-fed at 3 months. According to $24 \mathrm{~h}$ dietary recalls, we categorized 985 (45.2\%) infants as EBF at 3 months ( $47.8 \%$ among mothers with EBF intention; $31.7 \%$ among mothers with no EBF intention; $P<0.05)$ and $551(25.3 \%)$ infants as predominantly breast-fed at 3 months $(24.2 \%$ among mothers with EBF intention; $30 \cdot 8 \%$ among mothers with no EBF intention; $P<0 \cdot 05)$. Prenatal EBF intention was associated with $\mathrm{EBF}(\mathrm{OR}=1.48,95 \% \mathrm{CI} 1 \cdot 14,1.91)$ and with full breastfeeding $(\mathrm{OR}=1 \cdot 34,95 \% \mathrm{CI} 1.04,1.72)$ at age 3 months. EBF at age 3 months was not associated with maternal breast-feeding knowledge, attitudes or self-efficacy. Conclusions: Despite widespread expressed maternal EBF intention and universal breast-feeding initiation, prevalence of both exclusive and full breast-feeding at 3 months remains lower than WHO recommendations. EBF intention predicts breast-feeding behaviours, suggesting the importance of prenatal counselling to improve infant feeding behaviours.
\end{abstract}

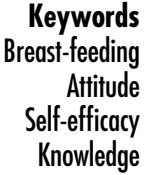

Keywords Attitude Knowledge
Globally, over $55 \%$ of infant deaths from diarrhoeal disease and acute respiratory infections are preventable by appropriate breast-feeding and complementary feeding ${ }^{(1)}$. Suboptimal infant feeding and endemic malnutrition contribute to mortality and morbidity, as well as to detrimental long-term developmental consequences, poorer educational performance and decreased productivity ${ }^{(2-7)}$.

Although the potential benefits of exclusive breastfeeding (EBF) through to infant age 6 months are widely recognized, EBF remains low in both developed and developing countries.

Maternal psychosocial factors and intentions to breastfeed have been associated, to varying extents, with infant feeding behaviours in previous literature. A nationally representative US study among 2372 women found that intention to partially or fully breast-feed predicted breastfeeding duration $(>6 \text { months })^{(8)}$. Similarly, in a cohort of 566 Australian women, intention to breast-feed was also positively associated with breast-feeding duration ${ }^{(9)}$. In separate study among 733 women, attitudes about benefits and barriers to breast-feeding, health-care systems, as well as social support were associated with breast-feeding initiation $^{(10)}$.

Over the past two decades, many studies have incorporated the Theory of Planned Behaviour (TPB), a general psychological model for human behaviour, as a theoretical framework to understand determinants of breast-feeding behaviours. According to the TPB, attitudes, subjective norms and perceived behavioural control predict intention to perform a certain behaviour; intention then predicts 
the behaviour ${ }^{(11,12)}$. In a meta-analytic review of 185 independent studies, $27 \%$ and $39 \%$ of the variance in behaviour and intention, respectively, were accounted for by the TPB framework ${ }^{(13)}$.

Among studies assessing maternal psychosocial risk factors relating to suboptimal breast-feeding, sample populations of women have been diverse, yet few are among women in under-resourced countries. Most studies focus on breast-feeding duration (attrition) and initiation; fewer centre on EBF.

Bangladeshi government policies and non-profit programmes have targeted inappropriate infant and young child feeding practices, addressing globally ranked malnutrition indicators (including the $41.3 \%$ of underweight children under 5 years of age ${ }^{(14)}$. Yet national prevalence of EBF through 6 months still has not improved for over a decade, despite nearly universal breast-feeding initiation ${ }^{(15)}$. There is a need to clarify the aetiology of early EBF attrition. Our objective was to further understand the associations between prenatal maternal psychosocial constructs, intentions and EBF behaviours at 3 months among women and their infants in rural north-eastern Bangladesh.

\section{Methods}

We conducted a prospective observational study embedded in a community-based nutrition and infant and young child feeding initiative (Akhoni Shomay - 'Window of Opportunity') implemented by CARE Bangladesh in Kishoreganj district, Bangladesh. In one sub-district (Karimganj), pregnant women and mothers with newborn infants were eligible to voluntarily participate in behaviour change communication activities and receive multiple-micronutrient supplementation. Women residing in a non-adjacent subdistrict (Katiadi) in the same district (Kishoreganj) were ineligible to participate in the CARE Bangladesh infant and young child feeding programme.

Located approximately $117 \mathrm{~km}$ from Dhaka, Karimganj and Katiadi are rural agrarian sub-districts with similar socio-economic and demographic characteristics. Approximately 320000 individuals in 70000 households reside in Karimganj. Katiadi has a population of 350000 individuals in 75000 households. Average household sizes are similar. Children under 5 years represent $17 \%$ of the total population in Karimganj and 15\% in Katiadi. Both sub-districts are located in the low-lying Haor region of north-eastern Bangladesh, which regularly experiences flooding and food insecurity. Several government and non-profit efforts, including the European Union's 'Food Security for the Ultra Poor' and CARE Bangladesh women's empowerment programmes, have centred on Karimganj.

\section{Study participants}

All field work for the present study was conducted by staff of icddr,b (International Centre for Diarrhoeal Disease
Research, Bangladesh). The study protocol approval was granted by the icddr,b Ethical Review Committee. Prior to study enrolment, eligible women were verbally informed about study objectives as well as protocol. Those who willingly signed participation consents were enrolled and interviewed. A team of twenty trained interviewers (eleven male; nine female) conducted the structured home interviews.

Study participants included mother-infant dyads in Karimganj and Katiadi sub-districts, from whom data were collected at approximately seven gestational months into pregnancy and 3 months after birth. Recruitment and enrolment of pregnant women occurred in six randomly selected unions (administrative units immediately above the level of the village) from each sub-district. In Bangladesh, subdistricts are comprised of unions; there are eleven total unions in Karimganj and ten in Katiadi. In each selected union, pregnant women were identified through the assistance of local non-profit organizations with programmatic foci on maternal and child health and that regularly monitor their respective areas for pregnancies. Women 15-49 years of age in their third trimester of pregnancy as computed based on the last menstrual period and permanently residing in the selected sub-districts were eligible. Exclusion criteria included residing for less than 6 months in Karimganj or Katiadi at study enrolment and any known or suspected chronic or congenital diseases. Women were interviewed during their third trimester of pregnancy in three waves, 4 months apart.

Of the 2400 women interviewed, 2397 gave birth to 2411 infants, including fourteen pairs of twins. The three remaining women were not pregnant or did not give birth within a biologically plausible time period. Losses to follow-up prior to interview at infant age 3 months included infant deaths, out-migration and refusal to further participate. Two thousand one hundred and seventy-eight (91\%) women completed the 3-month interview, achieving coverage of $>90 \%$ of pregnancies in the target communities.

Expecting regular seasonal migration of some Kishoreganj residents due to flooding and employment opportunities, interviewers maintained contact with study participants via cellular phones, scheduling home visits in advance as necessary. Nearly every study participant either owned or had access to a cellular phone.

\section{Data and variables}

Breast-feeding knowledge

We administered a six-item questionnaire to assess knowledge about optimal breast-feeding practices. We created a scale of breast-feeding knowledge (theoretical range 0-6) as the sum of the correct responses.

\section{Attitudes}

We used sixteen items from the Breastfeeding Attrition Prediction Tool (BAPT) to assess attitudes towards breastfeeding. Questions included nutrition impacts of EBF and 
inconveniences and difficulties of EBF for the mother. As interviewers in the first wave asked respondents to rate attitudes according to a scale of 1-3 and in the latter two waves to rate attitudes on a scale of $1-5$, we recoded wave 1 items as 1, 3 and 5. We reverse-coded negative-effect items before analysis. We used factor analysis with orthogonal (Kaiser) varimax rotation on the sixteen items and extracted three factors. In confirmatory factor analysis, fifteen of the sixteen attitudinal items had factor loadings $>0.5$. We created an overall index as a simple sum of scores for these fifteen items (theoretical range 15-75). Additional factor analysis identified three sub-constructs (time management - seven items; privacy concerns - three items; positive view of breast milk - five items); we computed indices for these sub-constructs as simple sums of item scores.

\section{Self-efficacy}

We used six items from the BAPT relating to self-efficacy and confidence of mothers regarding breast-feeding. All six self-efficacy questions were predictive of overall variance based on factor analysis loadings $(>0.65)$. We created an index as the simple sum of scores for these six items (theoretical range 6-30). Additional factor analysis identified two sub-constructs (emotional self-efficacy three items; objective self-efficacy - three items).

\section{Subjective norms}

We used five questions from the BAPT about perceived opinions of family members and health professionals regarding infant feeding. Due to lack of construct validity and a high proportion of non-responses, we did not develop an index of subjective norms.

\section{Breast-feeding intention}

The woman was asked how she intended to feed her infant. The responses were categorized as: EBF; breast milk with any other foods or liquids; no plan to provide breast milk; or no plans.

\section{Infant diet}

Interviews at 3 months included data collection on infant diet by $24 \mathrm{~h}$ and since-birth recalls. Liquid and food categories were derived from standard Bangladesh Demographic and Health Surveys (BDHS 2007) categories ${ }^{(16)}$. Reported breast-feeding practices (EBF, partial, full) were categorized according to UNICEF and WHO definitions ${ }^{(17)}$. Our definition of prelacteal feeds was similar to most other definitions, i.e. consumption of foods and liquids aside from breast milk (including honey, mustard oil) prior to EBF initiation. Although there is no consensus about the exact standard 'allowable' length of time after birth for prelacteals, in the present study we considered any non-breast milk feeds consumed during the first $3 \mathrm{~d}$ after birth as prelacteals.

\section{Statistical analysis}

Descriptive statistics were reported as means and standard deviations or proportions. Crude associations between categorical variables were assessed by Pearson's $\chi^{2}$ tests. We used logistic regression analyses to estimate associations between breast-feeding patterns at 3 months (based on $24 \mathrm{~h}$ recall) and prenatal intention, knowledge, attitudes and self-efficacy. We adjusted for covariates: maternal age (15-25, 26-35, > 36 years), previous births $(0,1,>1)$, socioeconomic status (quintiles based on household assets and durable goods), maternal literacy, any prenatal care for the current pregnancy and sub-district. Inclusion of these potential confounders was based on a priori knowledge from previous literature that establishes associations between each covariate separately with both psychosocial factors and breast-feeding behaviours. Additional potential covariates, specifically focusing on food security, were assessed; however, none were significant predictors of breast-feeding behaviours or confounders of any primary associations. Multivariate regressions were also stratified by sub-district. Analyses were performed using the statistical software package Stata release $10 \cdot 1$. With the final sample size of 2178 , the study had $90 \%$ power to detect differences (at $P<0.05$ ) of 10 percentage points between groups of women who did, or did not, intend to exclusively breastfeed their infants, assuming the observed prevalence of intention to $\mathrm{EBF}(85 \%)$ and the overall prevalence of EBF at 3 months ( $45 \%)$.

\section{Results}

On average, mothers were 24.8 (SD 5.6) years old. EBF intention was reported by $84.1 \%$ of mothers. Age did not differ by prenatal EBF intention (Table 1). A higher proportion of women in higher wealth quintiles reported EBF intention $(P<0.05)$. Higher proportions of women intending to $\mathrm{EBF}$ were literate $(67 \cdot 6 \%)$ relative to those not intending $(56.2 \% ; P<0.05)$. Accessing prenatal care during pregnancy was higher among women with EBF intention $(47 \cdot 1 \%)$, compared with those without $(32.6 \%$; $P<0.05)$.

Mean breast-feeding knowledge was 3.6 (SD 1.3). The mean maternal attitude regarding EBF was $55 \cdot 8$ (SD 8.2) and mean self-efficacy was 25.7 (SD 3.4). Prenatal attitudes and breast-feeding knowledge (both $P<0.05$ ), although not self-efficacy $(P>0.05)$, were significantly associated with maternal EBF intention.

\section{Infant feeding patterns}

During their first 0-3 d, many infants received prelacteal feeds (juices or high-sugar liquids $25.0 \%$, water $11.3 \%$, animal milk $2 \cdot 3 \%$, infant formula $2 \cdot 2 \%$ ). All mothers except one initiated breast-feeding and nearly all (99.6\%) mothers reported giving their infants breast milk in the previous $24 \mathrm{~h}$ (Table 2). At every time point ascertained 
Table 1 Selected characteristics of 2178 women recruited at 7 months' gestation and interviewed at 3 months postpartum, Kishoreganj District, rural north-eastern Bangladesh, by maternal prenatal exclusive breast-feeding (EBF) intention*

\begin{tabular}{|c|c|c|c|}
\hline & $\begin{array}{l}\text { EBF intention } \\
(n \text { 1831) }\end{array}$ & No EBF intention & $P$ \\
\hline Age (years) & & & 0.24 \\
\hline Mean & $24 \cdot 8$ & 24.4 & \\
\hline $\mathrm{SD}$ & $5 \cdot 6$ & $6 \cdot 0$ & \\
\hline \multicolumn{4}{|l|}{ Age group (\%) } \\
\hline $15-25$ years & $59 \cdot 8$ & $62 \cdot 8$ & 0.29 \\
\hline $26-35$ years & $35 \cdot 7$ & $32 \cdot 6$ & 0.27 \\
\hline$\geq 36$ years & 4.5 & 4.6 & 0.95 \\
\hline Number of previous births (\%) & & & $<0.01$ \\
\hline 0 & $25 \cdot 6$ & 34.0 & \\
\hline 1 & 25.4 & $17 \cdot 3$ & \\
\hline$>1$ & $49 \cdot 0$ & 48.7 & \\
\hline Literate $(\%)$ & 67.6 & $56 \cdot 2$ & $<0.01$ \\
\hline Any prenatal care during pregnancy† (\%) & $47 \cdot 1$ & 32.6 & $<0.01$ \\
\hline Household wealth index (\%) & & & 0.02 \\
\hline 1 (poorest) & 18.4 & 24.5 & \\
\hline 2 & $19 \cdot 7$ & $22 \cdot 8$ & \\
\hline 3 & 19.9 & 18.7 & \\
\hline 4 & $21 \cdot 1$ & $17 \cdot 0$ & \\
\hline 5 (least poor) & 20.9 & 17.0 & \\
\hline
\end{tabular}

*From interview at approximately 7 months' gestational age.

Table 2 Breast-feeding patterns* of 2178 children aged 3 months in Kishoreganj district, rural north-eastern Bangladesh, as obtained from $24 \mathrm{~h}$ recall and history of liquid consumption, by maternal prenatal exclusive breast-feeding (EBF) intention

\begin{tabular}{lcc}
\hline & $\begin{array}{c}\text { EBF intention } \\
(n \text { 1831) }\end{array}$ & $\begin{array}{c}\text { No EBF intention } \\
(n \text { 347) }\end{array}$ \\
\hline Intake in past 24 h (\%) & & 31.7 \\
Exclusively breast-fed & 47.8 & 30.8 \\
Predominantly breast-fed & 24.2 & 68.3 \\
Mixed feeding & 51.8 & 63.1 \\
Breast milk + other liquids & 48.9 & 1.2 \\
Breast milk + foods & 0.7 & 4.0 \\
Breast milk + foods + other liquids & 2.2 & 0.0 \\
No breast milk & 0.4 & 45.2 \\
Liquid consumption since birth (\%) & 55.9 & 26.2 \\
Only breast milk & 21.0 & 54.8 \\
Predominantly breast-feeding & 43.9 & 0.0 \\
Mixed feeding & 0.2 & \\
No breast milk & & \\
\hline
\end{tabular}

*EBF: not giving the infant any water or liquids aside from breast milk, or foods. Mixed feeding: breast milk is provided but is not the sole source of nourishment. Predominant breast-feeding: the predominant source of nourishment is breast milk although the infant may have received water and water-based drinks, fruit juice, oral rehydration salt solutions, drop and syrup vitamins, minerals, medicines and ritual fluids. Predominant breast-feeding is a subset of mixed feeding.

(0-3 d, $4 \mathrm{~d}, 2$ weeks, 1 month, 2 months and 3 months), $1 \%$ or fewer infants were not receiving any breast milk. Among infants not EBF at 3 months, the majority consumed other liquids (water $52.8 \%$, infant formula $12.4 \%$, animal milk $9.9 \%$, juices or high-sugar liquids $7.4 \%$ ), with fewer than $5.0 \%$ of infants consuming any soft, semi-solid or solid foods.

At 3 months, $45.2 \%$ of the infants were EBF and an additional $25 \cdot 3 \%$ were predominantly breast-fed (Table 2 ). Between birth and 3 months, EBF prevalence peaked at $4 \mathrm{~d}$ after birth (77.9\%; Fig. 1). A higher proportion of infants were EBF at 3 months among mothers reporting $\mathrm{EBF}$ intention $(47 \cdot 8 \%)$ compared with those without EBF intention $(31.7 \% ; P<0.05)$. Similarly at $4 \mathrm{~d}$ after birth, a higher proportion of infants of intending mothers (79.8\%) were $\mathrm{EBF}$, compared with those of non-intending mothers $(67.7 \% ; P<0.05)$. Conversely, the proportion of infants with non-intending mothers who were predominantly breast-fed at 3 months of age (30.8\%) was higher relative to those of intending mothers $(24.3 \% ; P<0.05)$.

\section{Prenatal maternal knowledge, attitudes, self- efficacy and intention in relation to breast-feeding at 3 months}

Mothers who reported EBF intention were more likely to $\mathrm{EBF}$ at 3 months (adjusted $\mathrm{OR}=1 \cdot 48 ; 95 \%$ CI 1.14, 1.91; 
Table 3, model 1). This association was robust to the addition of terms related to maternal knowledge, attitudes and self-efficacy (model 2); as well as in stratified analysis of one sub-district (Karimganj; see online supplementary material, Supplemental Table 1) but not the second subdistrict (Katiadi; Supplemental Table 2). Attitudes, selfefficacy and knowledge were not significantly associated with EBF at 3 months, whether considered on their own (Table 3, model 1), in joint models (Table 3, model 2) or stratified by sub-district (Supplemental Tables 1 and 2).

As $25.4 \%$ of infants were predominantly breast-fed, we additionally assessed full breast-feeding (defined as either exclusive or predominant) at 3 months as the dependent variable. EBF intention was associated with full breastfeeding at 3 months (OR $=1.34 ; 95 \%$ CI 1.04, 1.72) in the full model (Table 3, model 1). Maternal knowledge, attitudes and self-efficacy were not significantly associated with full breast-feeding at 3 months $(P>0.05)$ in the overall population (Table 3, model 2) or when stratified by sub-district (see online supplementary material, Supplemental Tables 1 and 2).

\section{Discussion}

\section{Breast-feeding initiation and exclusivity}

We observed universal breast-feeding initiation and approximately $45 \% \mathrm{EBF}$ at 3 months of age in rural Bangladesh. These estimates are similar to national statistics. According to the WHO Global Data Bank on Infant and Young Child Feeding, the national prevalence of breast-feeding initiation has remained over $96 \%$ since the $1990 \mathrm{~s}^{(15)}$; the national prevalence of EBF under 6 months is $42.9 \%^{(15)}$. It is difficult to compare EBF prevalence in our sample with national statistics as all infants in our sample were approximately 3 months old. As EBF prevalence generally declines as

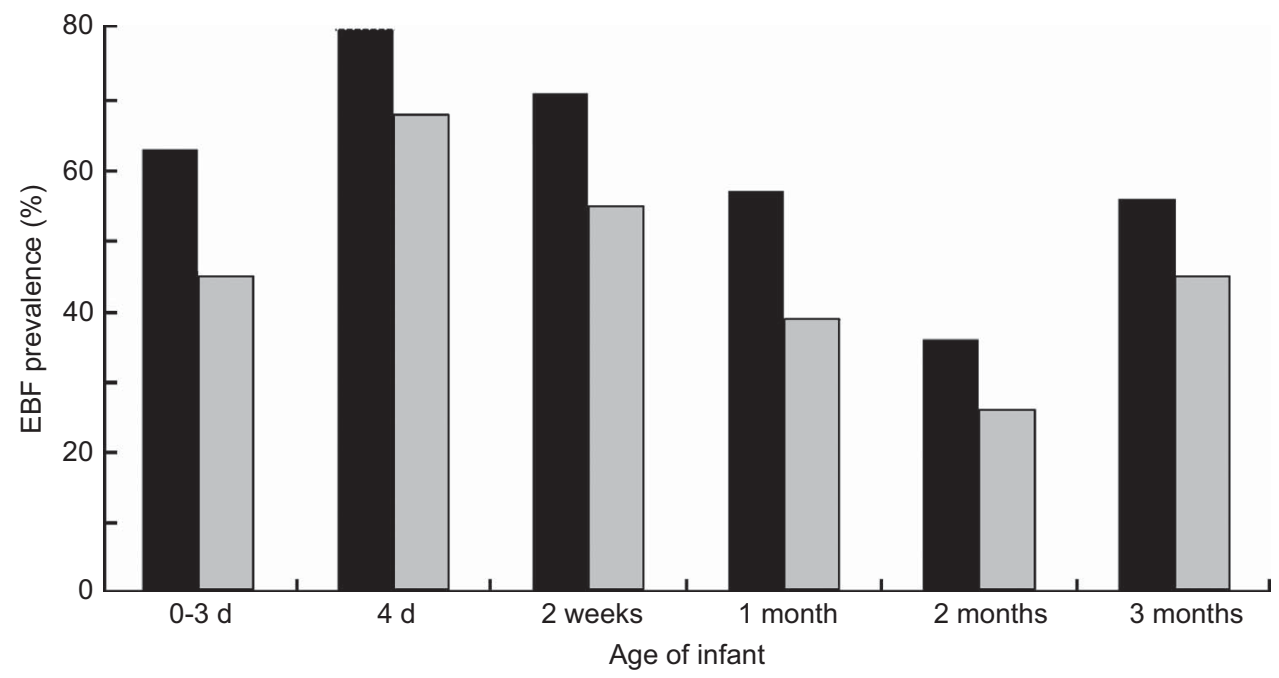

Fig. 1 Exclusive breast-feeding (EBF) prevalence at various ages from birth to 3 months among infants in Kishoreganj district, rural north-eastern Bangladesh, by maternal prenatal intention to exclusively breast-feed ( $\square$, EBF intention; $\square$, no EBF intention)

Table 3 Associations between breast-feeding pattern at 3 months and prenatal maternal intentions, attitudes, self-efficacy and knowledge among 2178 women interviewed during pregnancy and at infant age 3 months in Kishoreganj district, rural north-eastern Bangladesh

\begin{tabular}{|c|c|c|c|c|c|}
\hline \multirow[b]{2}{*}{ Outcome variable } & \multirow[b]{2}{*}{ Predictor variable } & \multicolumn{2}{|c|}{ Model $1^{*}$} & \multicolumn{2}{|c|}{ Model $2^{*}$} \\
\hline & & OR & $95 \% \mathrm{Cl}$ & OR & $95 \% \mathrm{Cl}$ \\
\hline \multirow[t]{4}{*}{ EBF at 3 months } & EBF intention† & 1.48 & $1.14,1.91$ & 1.43 & $1.10,1.85$ \\
\hline & Attitude towards breast-feeding $\ddagger$ & $1 \cdot 12$ & $0.94,1.34$ & 1.09 & $0.90,1.32$ \\
\hline & Self-efficacy $\ddagger$ & 1.01 & $0.84,1.21$ & 0.99 & $0.82,1.20$ \\
\hline & Breast-feeding knowledge & 1.22 & $1.00,1.50$ & $1 \cdot 17$ & $0.95,1.44$ \\
\hline \multirow[t]{4}{*}{ Full breast-feeding at 3 months } & EBF intention† & 1.34 & $1.04,1.72$ & 1.32 & $1.02,1.70$ \\
\hline & Attitude towards breast-feeding & 1.06 & $0.88,1.28$ & 1.03 & $0.85,1.26$ \\
\hline & Self-efficacył & 1.01 & $0.84,1.23$ & 1.02 & $0.83,1.24$ \\
\hline & Breast-feeding knowledge & $1 \cdot 14$ & $0.92,1.42$ & $1 \cdot 10$ & $0.88,1.38$ \\
\hline
\end{tabular}

EBF, exclusive breast-feeding.

*Model 1, each predictor tested separately; Model 2, all predictors entered into the model simultaneously. All models additionally control for maternal age, previous births, household wealth quintile, maternal literacy, prenatal care during pregnancy and sub-district.

†Maternal EBF intention assessed at 7 months' gestational age.

łlndices for maternal attitudes (from fifteen items), self-efficacy (six items) and knowledge (six items) based on simple sums of relevant questions, according to factor analysis. Continuous maternal indices dichotomized at median values. 
infants age, this suggests that EBF prevalence in Karimganj and Katiadi may be lower than the national average.

In the past two decades, national surveys have reported a wide range of EBF prevalence $(36 \cdot 2-58 \cdot 4 \%)^{(15)}$. Although current national recommendations are to EBF until 6 months, previous recommendations in the early 2000s were only until 5 months of age ${ }^{(18)}$. This modified definition likely accounts for some variability of reported EBF prevalence.

Our multivariate regression models reflected a theoretical model derived from the TPB, which hypothesizes that attitudes and self-efficacy drive EBF intention, which translates to behaviours. We modified the classic TPB model by excluding maternal subjective norms due to lack of construct validity in our sample and we included a component of breast-feeding knowledge. While this limits direct comparisons with studies that incorporate all three psychosocial indices in the TPB, our observations are consistent with the TPB conceptual framework in general. We previously showed that maternal prenatal breast-feeding attitudes and self-efficacy were positively associated with EBF intention during the third trimester of pregnancy ${ }^{(19)}$. Our present findings indicate associations between EBF intention and postnatal breast-feeding behaviours, but weaker associations between the underlying prenatal constructs and actual behaviour. Further research is needed to assess intention, psychosocial factors and breast-feeding behaviours while accounting for subjective norms, in a rural Bangladesh context.

Our results suggest that some, but not all, of the psychosocial factors we assessed were predictive of EBF intention among rural Bangladeshi women, which is similar to previous studies in other settings that consider attitudes ${ }^{(20,21)}$ and knowledge ${ }^{(22)}$ in relation to breast-feeding intentions. Prenatal attitudes and self-efficacy were not predictive of EBF or full breast-feeding at 3 months of age. On average, women had relatively strong self-efficacy (26.6 on a $0-30$ scale); the weak predictive power of breast-feeding behaviours may be related to the low variability (SD 3.4). Earlier studies found that attitudinal indices did not predict breastfeeding duration ${ }^{(23,24)}$ or exclusivity ${ }^{(25)}$. Some literature suggests that maternal confidence is not predictive of breastfeeding behavioural outcomes ${ }^{(10,23,24,26)}$. Similarly, a study among 599 Jamaican women found no influence of maternal knowledge regarding breast-feeding on $\mathrm{EBF}^{(25)}$.

On the other hand, many studies indicate that attitudes $^{(9,20,21,26-34)}$, self-efficacy ${ }^{(21,27,28,31-33,35-38)}$ and knowledge $e^{(10,22,39)}$ are predictive of breast-feeding initiation and duration. Differences from our results could stem from difficulties in comparing across disparate breastfeeding behavioural outcomes, since we report on EBF and full breast-feeding rather than initiation and attrition. Additionally, socio-economic differences need to be accounted for as the majority of studies were among women residing in better-resourced (primarily US) settings.

Compared with other psychosocial factors (attitudes, self-efficacy, subjective norms), relatively fewer studies have assessed the extent to which intention to breast-feed predicts infant feeding behaviours of mothers. Among relevant studies, all found positive associations ${ }^{(8,26,36,40)}$, which aligns with our study findings.

Unlike studies that ascertain maternal intentions retrospectively, our study was prospective, eliminating the possibility that the woman's responses are altered by future behaviour. Our study had high follow-up through age 3 months, which likely limits participation bias. Efforts were made to minimize cognitive comprehension issues during interviews by acknowledging issues identified in previous literature about differing interpretations of EBF definition and duration ${ }^{(38,41)}$. Interviewers asked simple, direct questions about foods and liquids consumed, as well as specified exact times in both days and months. EBF and full breast-feeding categories were created during analysis based on dietary intake, according to consistent breast-feeding definitions.

The survey-based data collection was a potential source of self-report bias and recall bias. Women may have under-reported negative perceptions and behaviours. Accuracy of self-reported dietary recalls relies on mothers correctly remembering the dietary consumption of their infants at specified times prior to the interview.

Despite high expressed maternal intentions to EBF during pregnancy and universal breast-feeding initiation, prevalence of both exclusive and full breast-feeding at 3 months remain lower than WHO recommendations. Our findings indicate associations between EBF intention and postnatal breastfeeding behaviours, suggesting the importance of increasing activities to raise levels of prenatal EBF intention to support optimal infant feeding behaviours.

\section{Acknowledgements}

Acknowledgments: This study was embedded in CARE's community-based initiative to improve infant and young child feeding and nutritional outcomes, known as Akboni Shomay or 'Window of Opportunity', in Bangladesh. The authors would like to thank the field staff and team leads in Bangladesh; the staff of icddr,b and CARE Bangladesh; and the women for their participation. Financial support: This study was supported by a grant from CARE USA (000013123). The authors have no financial relationships relevant to this article to disclose. Conflict of interest: None. Authorship: A.D.S., B.S., S.R. and A.S.G.F. designed the cohort study. A.S.G.F. and S.K.D. supervised the data collection and led field activities. E.A.Y., A.D.S. and B.S. conceived and planned the statistical analysis. E.A.Y., J.S.T., A.O. and N.T. analysed the data. E.A.Y. and A.D.S. wrote the manuscript. All authors critically reviewed the manuscript for important intellectual content and reviewed and approved the final version. Ethics of human subject participation: The study was approved by the Ethical Review Committee of icddr,b. 


\section{Supplementary material}

To view supplementary material for this article, please visit http://dx.doi.org/10.1017/S1368980014000937

\section{References}

1. World Health Organization \& UNICEF (2003) WHO/UNICEF Global Strategy for Infant and Young Child Feeding. Geneva: WHO.

2. Horta BL, Bahl R, Martines JC et al. (2007) Evidence on the Long-Term Effects of Breastfeeding: Systematic Review and Meta-Analyses. Geneva: WHO.

3. Ip S, Chung M, Raman G et al. (2007) Breastfeeding and Maternal and Infant Health Outcomes in Developed Countries. Evidence Report/Technology Assessment no. 153. Rockville, MD: Agency for Healthcare Research and Quality.

4. Arifeen S, Black RE, Antelman G et al. (2001) Exclusive breastfeeding reduces acute respiratory infection and diarrhoea deaths among infants in Dhaka slums. Pediatrics 108, E67.

5. Victora CG, Smith PG, Vaughan JP et al. (1989) Infant feeding and deaths due to diarrhea: a case-control study. Am J Epidemiol 129, 1032-1041.

6. Dewey KG, Cohen RJ, Brown KH et al. (2001) Effects of exclusive breastfeeding for four versus six months on maternal nutritional status and infant motor development: results of two randomized trials in Honduras. J Nutr 131, 262-267.

7. Kramer MS, Guo T, Platt RW et al. (2003) Infant growth and health outcomes associated with 3 compared with 6 mo of exclusive breastfeeding. Am J Clin Nutr 78, 291-295.

8. Piper S \& Parks PL (1996) Predicting the duration of lactation: evidence from a national survey. Birth 23, 7-12.

9. Scott JA, Binns CW, Oddy WH et al. (2006) Predictors of breastfeeding duration: evidence from a cohort study. Pediatrics 117, e646.

10. Khoury AJ, Moazzem SW, Jarjoura CM et al. (2005) Breastfeeding initiation in low-income women: role of attitudes, support, and perceived control. Womens Health Issues 15, 64-72.

11. Ajzen I (1991) The theory of planned behavior. Organ Behav Hum Decis Process 50, 179-211.

12. Ajzen I (2002) Perceived behavioral control, self-efficacy, locus of control, and the theory of planned behavior. $J$ Appl Soc Psychol 32, 665-683.

13. Armitage C \& Conner M (2001) Efficacy of the theory of planned behavior: a meta-analytic review. Br J Soc Psychol 40, 471-499.

14. World Health Organization (2011) World Health Statistics 2011. Geneva: WHO.

15. World Health Organization (2010) WHO Global Data Bank on Infant and Young Child Feeding (IYCF): Bangladesh. Geneva: WHO.

16. National Institute of Population Research and Training, Mitra and Associates \& Macro International (2009) Bangladesh Demographic and Health Survey 2007. Dhaka and Calverton, MD: NIPORT, Mitra and Associates and Macro International.

17. World Health Organization (2008) Indicators for Assessing Infant and Young Child Feeding Practices (Part I: Definitions): Conclusions of A Consensus Meeting Held 6-8 November 2007 in Washington D.C., USA. Geneva: WHO.

18. Haider R, Ashworth A, Kabir I et al. (2000) Effect of community-based peer counsellors on exclusive breastfeeding practices in Dhaka, Bangladesh: a randomised controlled trial. Lancet 356, 1643-1647.

19. Thomas JS, Yu EA, Tirmizi N et al. (2014) Maternal knowledge, attitudes and self-efficacy in relation to intention to exclusively breastfeed among pregnant women in rural Bangladesh. Matern Child Health J (Epublication ahead of print version).

20. Gill SL, Reifsnider E, Lucke JF et al. (2007) Predicting breastfeeding attrition: adapting the breast-feeding attrition prediction tool. J Perinat Neonatal Nurs 21, 216-224.

21. Wambach K (1997) Breastfeeding intention and outcome: a test of the theory of planned behavior. Res Nurs Health 20, 51 .

22. Mitra AK, Khoury AJ, Hinton AW et al. (2004) Predictors of breastfeeding intention among low-income women. Matern Child Health J 8, 65-70.

23. Evans ML, Dick MJ, Lewallen LP et al. (2004) Modified breastfeeding attrition prediction tool: prenatal and postpartum tests. J Perinat Educ 13, 1-8.

24. Lewallen LP, Dick MJ, Wall Y et al. (2006) Toward a clinically useful method of predicting early breast-feeding attrition. Appl Nurs Res 19, 144-148.

25. Chatman L, Hasumi S, Roofe M et al. (2004) Influence of knowledge and attitudes on exclusive breastfeeding practice among rural Jamaican mothers. Birth 31, 265-271.

26. Lawson K \& Tulloch MI (1995) Breastfeeding duration: prenatal intentions and postnatal practices. J Adv Nurs 22, 841-849.

27. Dick MJ, Evans ML, Arthurs JB et al. (2002) Predicting early breastfeeding attrition. J Hum Lact 18, 21-28.

28. Mossman M, Heaman M, Dennis CL et al. (2008) The influence of adolescent mothers' breastfeeding confidence and attitudes on breastfeeding initiation and duration. J Hum Lact 24, 268-277.

29. De La Mora A \& Russell DW (1999) The Iowa Infant Feeding Attitude Scale: analysis of reliability and validity. $J$ Appl SOC Psychol 29, 2362-2380.

30. Leff EW, Jefferis SC \& Gagne MP (1994) The development of the maternal breastfeeding evaluation scale. J Hum Lact $\mathbf{1 0}$, 105-111.

31. Janke JR (1992) Prediction of breast-feeding attrition: instrument development. Appl Nurs Res 5, 48-53.

32. Janke JR (1994) Development of the breast-feeding attrition prediction tool. Nurs Res 43, 100-104.

33. O'Campo P, Faden RR, Gielen AC et al. (1992) Prenatal factors associated with breastfeeding duration: recommendations for prenatal interventions. Birth 19, 195-201.

34. Ekwo EE, Dusdieker LB, Booth B et al. (1984) Psychosocial factors influencing the duration of breastfeeding by primigravidas. Acta Paediatr 73, 241-247.

35. Blyth R, Creedy D, Denis C et al. (2002) Effect of maternal confidence on breastfeeding duration: an application of breastfeeding self-efficacy theory. Birth 29, 278-284.

36. Dodgson JE, Henly SJ, Duckett L et al. (2003) Theory of planned behavior-based models for breastfeeding duration among Hong Kong mothers. Nurs Res 52, 148-158.

37. Duckett L, Henly S, Avery M et al. (1998) A theory of planned behavior based structural model for breast-feeding. Nurs Res 47, 325-335.

38. Dennis CL (2003) The breastfeeding self-efficacy scale: psychometric assessment of the short form. J Obstet Gynecol Neonatal Nurs 32, 734-744.

39. Wambach KA, Aaronson L, Breedlove G et al. (2011) A randomized controlled trial of breastfeeding support and education for adolescent mothers. West J Nurs Res 33, 486-505.

40. Janke JR (1988) Breastfeeding duration following cesarean and vaginal births. J Nurse Midwifery 33, 159-164.

41. Haider R, Kabir I \& Ashworth A (1999) Are breastfeeding promotion messages influencing mothers in Bangladesh? Results from an urban survey in Dhaka, Bangladesh. J Trop Pediatr 45, 315-318 